\title{
Design of an Electronic Upload and Reporting System aimed at Corelab Tasks and Responsibilities in Multi-center Clinical Trials
}

\author{
Jan Walter Benjamins, Yoran M Hummel, Jan Peter Busman, Frans Riepma, Bernard Dorhout, \\ Joost P van Melle \\ Groningen Imaging Core Laboratory, University Medical Center Groningen, University of \\ Groningen, Groningen, The Netherlands
}

\begin{abstract}
In multicenter clinical trials images are interpreted and analyzed by an imaging core laboratory (ICL) to generate consistent results with the least possible variance.

Currently, transfer of the images can be achieved via postal services (physical media) or web-based via upload portals that merely act as a digital substitute for traditional logistics. Image verification before and data entry after analysis is performed manually with possible introduction of human errors.

We describe the design of a software system that facilitates general ICL logistics, such as digital transfer of images, automated DICOM data verification, semiautomated image quality assessment, reporting and automated database generation in multicenter clinical trials.

All steps in the workflow are guided by and validated against the trial design. Validation failure(s) at any point are reported instantaneously to all persons concerned, consequently increasing efficiency and possibly reducing trial costs.
\end{abstract}

\section{Introduction}

In multi-center cardiovascular trials utilization of Imaging Core Laboratories (ICLs) is advised to generate consistent results with the least possible variance [1].

In general these trials are organized as follows. 1. Image acquisition (on site), 2. Image transfer (between site and ICL), 3. Image verification/quality check (in ICL), 4. Image analysis (by ICL), 5. Data entry (by ICL), 6. Results transfer (by ICL to Sponsor).

Traditionally, images carried on physical media, accompanied by corresponding paper forms are transferred to ICLs via postal services. During and after upload of the images to the ICL Medical Imaging System (MIS), technical, administrative and qualitative image verification are performed manually by a Quality
Assurance (QA) officer at the ICL. If any of the verifications fail, the delivering site and the supporting Contract Research Organization (CRO) are contacted by the ICL. Image analysis is executed by ICL technicians on echo workstations. Data entry is performed manually by ICL staff on (e)CRF (CRF = Case Report Form). Taken as a whole, the described workflow is time consuming and prone to error.

More recently CROs have set up web-based upload portals to confine some of the delay associated with transfer via postal services, but these portals act merely as a digital substitute for the traditional logistics. Digital documents accompanying the uploaded images are unstructured and since there is no application that verifies or could verify their contents, human verifications are still required.

In the search for alternatives that better suit an ICL's workflow, we found some solutions that focused more on electronic data capture (EDC) in clinical trials [2] or on reuse of clinical data for research [3,4] rather than on integration with an ICL's MIS. In addition, analysis on dedicated echo workstations and QA-operations regarding the images are beyond the scope of these solutions. Finally, none of the investigated alternatives provides any appliance to verify the images against trial setup.

In conclusion, we saw the need to develop a customized system aiming at the ICL specific tasks in multicenter trials and focusing on ICL responsibilities to further improve and optimize the way ICLs participate in these trials.

\section{Objective}

We describe the design of an electronic Imaging Corelab Workflow System (ICWS), developed by Groningen Imaging Core Laboratory (www.g-icl.com), that enhances image transfer, image verification and generation of results in multicenter cardiovascular clinical trials.

The described design follows guidance provided by earlier work about ICL best practices in clinical trials [1]. 
Even though an image-server is beyond the scope of the ICWS, technical requirements of the ICWS rely on the assumption that a general image-server complies with DICOM 3 and HL7V2 standards and is equipped with DICOM Store functionality as well as an HL7 service which broadcasts unsolicited observation result (ORU) messages.

\subsection{Requirements}

R1. The application should take care of secure transport and storage of the images, uploaded by sites.

R2. The application should provide an intuitive interface accessible to sites to enter administrative data and upload the DICOM images acquired on site.

R3. The application should serve automated image upload to the ICL's MIS hosted on the ICL intranet.

R4. The application should fully automate image verification to minimize delays, human errors and reduce personnel costs.

R5. QA Tasks should be fully integrated into the system and automated as much as possible.

R6. To increase general trial performance and reduce delay of results, the system should provide direct feedback to anyone involved and configured to the trial sponsors' requirements.

R7. The application should provide automated collection of measurement results broadcasted by the ICL's MIS.

\section{Methods}

\subsection{Architecture}

To create a system that meets requirements R2 and R3, a dual system was chosen. A web application is set up in the ICL Demilitarized Zone (DMZ) to provide site users with a tool to register study details in a web form and subsequently upload DICOM images from their local storage without jeopardizing the privacy of data within the ICL. Transport Layer Security (TLS) is used for secure data transfer between the DMZ server and the clients (R1).

The other part of the ICWS is set up in the ICL intranet, where an intranet based web application provides QA-officers with the tools required to inspect uploaded images and to upload images into the ICL ultrasound archive over a DICOM network connection. In addition, the intranet application provides system administration tools to set up clinical trial configurations. Another application component is installed on the intranet server to collect measurement results from the image server by serving an HL7 ORU (Observation Results) service.

All system components have been developed in ASP.NET. Both web applications are implemented in ASP.NET MVC. The ORU-service is based on Windows Communication Foundation (WCF) technology.

The two applications share an application database containing all trial configurations and all data concerning studies, uploads and measurement results - and a file share where uploaded images can be stored. The database and file share are located on the ICL's intranet with access limited to both application servers. For security reasons, because the DMZ server is accessible from the internet, the server is completely isolated from the ICL intranet aside from these two data connections (R1).

\subsection{Authentication}

Access to the DMZ based web site is secured with two factor authentication, based on industry standard algorithms for time-based one-time password (TOTP) authentication [5,6], implemented by the Microsoft Authenticator and Google Authenticator apps. On the intranet web application ICL users are authenticated by their network credentials.

\subsection{Image transfer and verification}

Following login site users can start a new upload in the internet based application by entering a short registration form. Since site and user information are known by the system based on the login, a predefined minimal set of data needs to be entered by the user. The user selects the sonographer, echo-scanner machine model, date of echo and enters a subject number. Based on trial configuration additional information (i.e. gender, date of birth) can be made mandatory. The user is guided while entering the form fields. For each field the user is shown whether a value is mandatory and in case of a text field, information about the predefined formatting is displayed.

After submitting the registration form, the user can select files on his local disk or available network shares and start his upload. File size is limited to the server application's technical configuration, which is therefore scaled to a proportion that guarantees upload of current multi-frame images. For each file a 256bit SHA hash [7] is calculated on the client computer, which is sent together with the file contents to the server. While the next upload is in progress, the uploaded file's integrity is verified by calculating the file-hash after upload and comparing it to the client's hash value. If the hashes match, the file is passing a set of server side validations. The service checks whether the uploaded file is a DICOM file, the modality is ultrasound (US) and calibration data is available, which is a requirement to perform measurements on an ultrasound workstation. If these 
checks are passed, the file is considered technically correct and the applications proceeds with further administrative checks. An exact match with the detail provided in the digital registration forms is required. Furthermore, general and trial specific rules regarding depersonalization are verified. If any of the validation steps fails, file upload is aborted and the user is provided on screen with the reason of failure.

If all files have passed the validation, the user can either choose to select an additional set of files or explicitly mark the upload as completed. At this point, the system has verified whether the uploaded files satisfy the conditions to be uploaded to the archive (R4).

\subsection{Automated feedback}

In addition to on screen information about validation errors, a configured set of people participating in the trial

Site
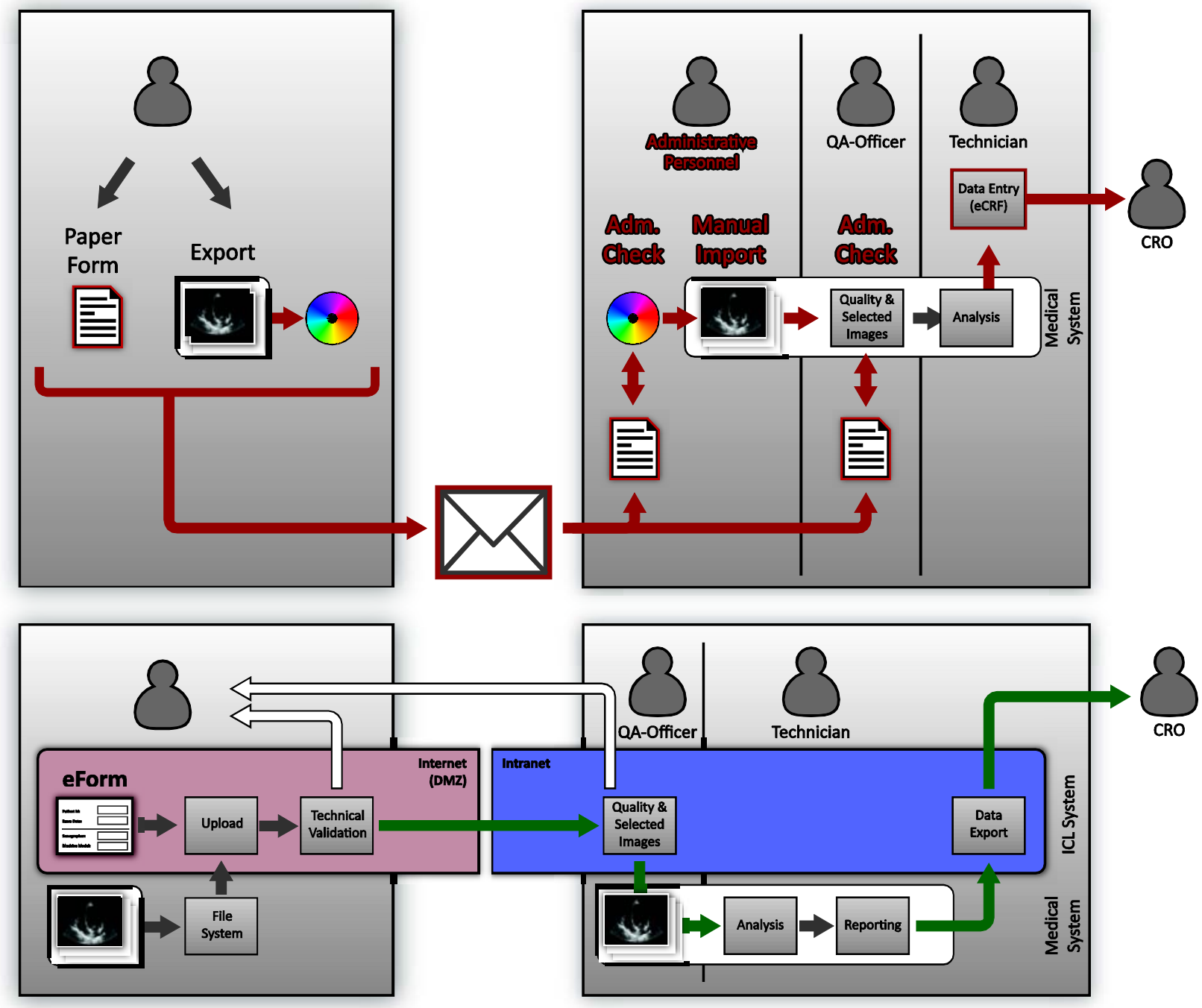

Figure 1- Comparison of the designed system (below) to the original process (above)
- being ICL, CRO or site personnel - can be informed with the event of a failed upload via email. This applies to each step in the workflow of the system; if anything happens with an upload at any given point in the process that one or more participants need to be informed about, the system can be configured to send an email instantly and for each event a specific set of recipients can be configured. This is of course based on the sponsor's requirements (R6).

\subsection{Image verifications / $\mathrm{QA}$ checks}

After the site has completed file uploads for a study, the study becomes visible to the ICL's QA-personnel in the ICL intranet application (R5). A QA-Officer is notified on newly received exams and verifies, whether all images required for the corresponding trial have been uploaded, image quality is sufficient and corresponding

$\mathrm{ICL}$

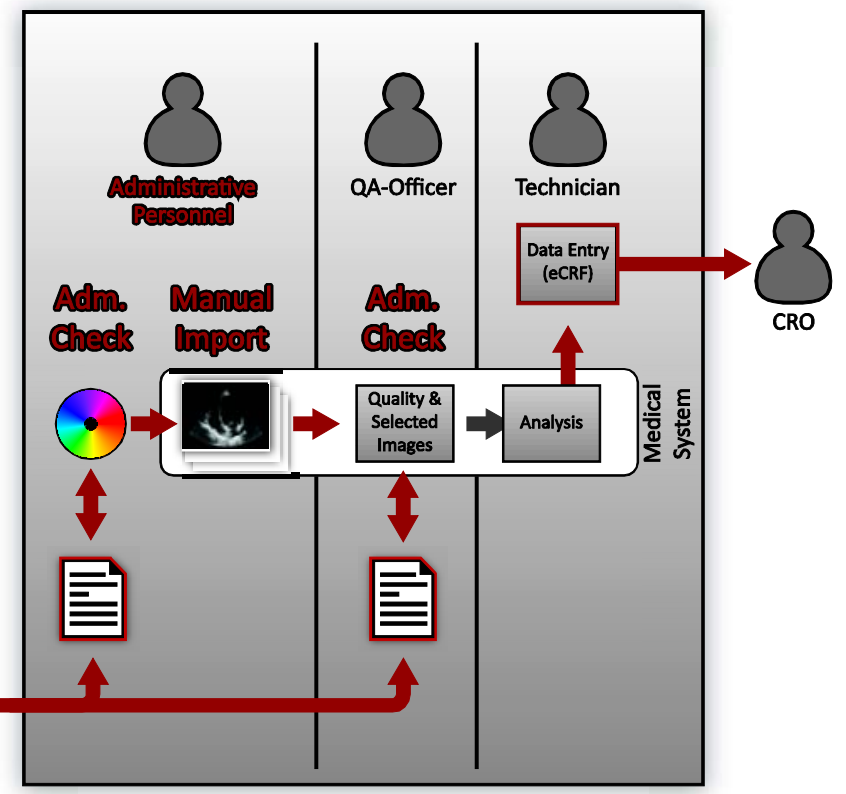


documentation is fully matching. The QA may choose to reject the upload if, from a qualitative perspective, the upload does not meet general ICL or trial specific requirements.

On approval of the upload, the system sends the images to the ICL's image server over a DICOM network connection (R3), where the study and its images are available to the ICL's echo workstations. All verifications have taken place before the files are uploaded to the ultrasound archive within the least possible time and anyone who may be concerned with the fact corrections need to be made, has been informed instantly.

\subsection{Generation and transfer of results}

Measurements are performed on echo workstations as before. The results are sent back from the image server to the ICL system via an HL7 connection as an observation results (ORU) message (R7). The received message is traced back to the corresponding study registration and clinical trial. If the measurements have been stored on the echo workstation by a technician that was admitted to the trial, measurements are verified against trial configuration. If all predefined measurements are present in the HL7 message, the technician's set of measurements is marked as completed. Otherwise, the technician is sent an email containing a statement which additional measurement are required.

A trial may be configured with single or multi observers, analyzing the images. When a complete set of measurements has been received by the HL7 service, the application verifies, whether a sufficient number of technicians have submitted their measurements. In that case, the study registration is marked as completed.

From all completed trial studies results can be generated automatically or manually and sent to the CRO or the trial sponsor.

\section{Discussion and conclusions}

The provided system can replace unnecessary human interactions in ICLs' trial workflows with automated software components (figure 1). The system meets the set requirements. We foresee reduced delay of image analysis results and costs running a clinical trial. Furthermore, with the introduction of the system, ICLs should be able to participate in more clinical trials without increase of administrative overhead. However, as the system is used and while the digital working environment develops, new ideas and requirements may arise.

The system was designed and built based on generally accepted and adopted medical communication standards, but with a single MIS implemented. When advanced measurements such as 2D strain echocardiography are included in a clinical trial, depending on the brand of the ultrasound scanners used for image acquisition, multiple vendors' software systems may be required to analyze all collected images in a single clinical trial. Therefore, we consider to extend the system with support for trial based configuration of one or more target image servers.

In addition, since the mentioned medical standards have been implemented, we could extend the system's focus from echocardiography to other imaging modalities.

Compared to the investigated alternatives, the described application design much better suits the needs of an ICL. One of the alternatives, however, was built around the idea of automatically feeding an EDC system with clinical trial data [2]. Adding such functionality to the end of the application workflow, would enhance the system.

\section{References}

[1] Douglas PS, DeCara JM, Devereux RB, Duckworth S, Gardin JM, Jaber WA, et al. Echocardiographic imaging in clinical trials: American Society of Echocardiography Standards for echocardiography core laboratories: endorsed by the American College of Cardiology Foundation. J Am Soc Echocardiogr 2009;22(7):755-765.

[2] Haak D, Page CE, Reinartz S, Kruger T, Deserno TM. DICOM for Clinical Research: PACS-Integrated Electronic Data Capture in Multi-Center Trials. J Digit Imaging 2015;28(5):558-566.

[3] El Fadly A, Daniel C, Bousquet C, Dart T, Lastic PY, Degoulet P. Electronic Healthcare Record and clinical research in cardiovascular radiology. HL7 CDA and CDISC ODM interoperability. AMIA Annu Symp Proc 2007:216-220.

[4] El Fadly A, Rance B, Lucas N, Mead C, Chatellier G, Lastic PY, et al. Integrating clinical research with the Healthcare Enterprise: from the RE-USE project to the EHR4CR platform. J Biomed Inform 2011;44 Suppl 1:S94102.

[5] D. M'Raihi, M. Bellare, F. Hoornaert, D. Naccache, O. Ranen. HOTP: An HMAC-Based One-Time Password Algorithm. December 2005.

[6] M'Raihi D, Machani S, Pei M, Rydell J. TOTP: TimeBased One-Time Password Algorithm. May 2011.

[7] U.S. Department of Commerce, National Institute of Standards and Technology. Secure Hash Standard (SHS). August 2015.

Address for correspondence:

Jan Walter Benjamins

University Medical Center Groningen

Department of Cardiology

P.O. Box 30.001

9700RB Groningen, The Netherlands

j.w.benjamins@umcg.nl 